\title{
Solution of the Initial Value Problem for the sine-Gordon Equation Using a Kac-Moody Algebra
}

\author{
Paul Mansfield *
}

Department of Applied Mathematics and Theoretical Physics, University of Cambridge, Silver Street, Cambridge CB3 9EW, United Kingdom

\begin{abstract}
We solve the classical sine-Gordon equation using a Lax pair belonging to a Kac-Moody algebra. By realising the algebra in terms of fermionic currents we reduce the initial value problem to the evaluation of a fermionic propagator as a sum of Feynman diagrams.
\end{abstract}

\section{Introduction}

The sine-Gordon equation,

$$
\frac{\partial^{2} \varrho}{\partial x^{2}}-\frac{\partial^{2} \varrho}{\partial t^{2}}=\sin \varrho
$$

is a relativistic equation in one space and one time dimension with many applications in mathematical physics [1]. Soliton solutions have been constructed using the inverse scattering transform [2]. Here we describe a different approach, based on a Kac-Moody algebra, which solves the equation for arbitrary initial data. The method reveals an intimate relationship between solutions of the sineGordon equation and the propagator for a certain fermionic quantum field theory. This is a precursor at the classical level of the well-known equivalence between the quantum sine-Gordon theory and the massive Thirring model [3].

The starting point for the solution of the sine-Gordon equation by the inverse scattering transform is its associated Lax pair. Conventionally, this is constructed from elements of the Lie algebra sl $(2, \mathbb{C})$. Leznov and Smirnov [4] have pointed out that the Kac-Moody algebra may be used instead. The advantage of this is that the same construction that was used to solve the Toda equations by Leznov and Saveliev [5] may be exploited to relate solutions of the sine-Gordon equation to elements of the Kac-Moody group constructed from initial data given on some light-cone. Jimbo and Miwa have also constructed solutions using the KacMoody algebra [8]. In Sect. 2 we give a different derivation of this construction

* Address from 1 October 1984: CERN, Geneva 
which allows us to specify the initial data on a spacelike surface. A similar result was obtained by Onofri for the Liouville equation [6]. At this stage the solution appears quite formal because the Kac-Moody algebra is infinite and the group elements remain to be evaluated.

In Sect. 3 we describe a fermionic representation of the Kac-Moody algebra that enables us to evaluate the group elements using Wick's theorem. Thus the solution of the sine-Gordon equation reduces to the evaluation of a two-point function which has the usual diagrammatic expansion, and depends only on the initial data. The final section is a discussion of similarities between our work and the Riemann Transform method which is a formulation of the Inverse Scattering Transform due to Shabat, Zakharov, and Mikhailov [7]. In an Appendix we give details of the fermionic representation of the Kac-Moody algebra.

\section{Solution in Terms of the Kac-Moody Algebra}

In this section we define a Lax pair for the sine-Gordon equation in terms of a KacMoody algebra, and exploit this to express the sine-Gordon field as a function of an element of the corresponding Kac-Moody group depending only on the initial data. We begin by describing the Kac-Moody algebra.

Let $\left\{L^{i} ; i=1 \ldots D\right\}$ be a set of $n \times n$ matrices with the Lie algebra commutation relations

and Killing form

$$
\left[L^{i}, L^{j}\right]=f^{i j k} L^{k},
$$

$$
\eta^{i j}=\operatorname{Tr}\left(L^{i} L^{j}\right) \text {. }
$$

The generators of the corresponding infinite dimensional Kac-Moody algebra [9] carry a subscript which takes on integral values and satisfy the commutation relations

$$
\left[L_{m}^{i}, L_{n}^{j}\right]=f^{i j k} L_{m+n}^{k}+m \delta_{m,-n} \eta^{i j} \mathbb{1} .
$$

$\mathbb{1}$ is a central element which commutes with the $L_{n}^{i}$. If we take as generators of $\operatorname{sl}(2, \mathbb{C})$ [the complexification of $\mathrm{SU}(2)]$ the following combinations of Pauli matrices

$$
H=\sigma_{3}, \quad E^{ \pm}=\frac{1}{2}\left(\sigma_{1} \pm i \sigma_{2}\right),
$$

then from (3) the corresponding Kac-Moody algebra is

$$
\begin{aligned}
{\left[H_{m}, H_{n}\right] } & =2 m \delta_{m,-n} \mathbb{1}, \\
{\left[H_{m}, E_{n}^{ \pm}\right] } & = \pm 2 E_{m+n}^{ \pm}, \\
{\left[E_{m}^{ \pm}, E_{n}^{ \pm}\right] } & =0, \\
{\left[E_{m}^{+}, E_{n}^{-}\right] } & =H_{m+n}+m \delta_{n,-m} \mathbb{1} .
\end{aligned}
$$

We can now specify the Lax pair. Define the fields

$$
\mathscr{A}_{ \pm}= \pm \frac{1}{4} i \partial_{ \pm} \varrho H_{0} \pm \partial_{ \pm} \phi \mathbb{1}+\alpha e^{i \varrho / 2} E_{0}^{ \pm}+\alpha e^{-i \varrho / 2} E_{ \pm 1}^{\mp} .
$$

Here $\varrho$ is a real function and $\phi$ a complex function of space-time and

$$
Z^{ \pm}=\frac{1}{2}(t \pm x), \quad \partial_{ \pm} \equiv \frac{\partial}{\partial Z^{ \pm}}, \quad \partial^{2}=\partial_{+} \partial_{-} .
$$


We now impose the condition that $\mathscr{A}$ be a pure gauge, that is that there exists an element of the Kac-Moody group $g$ such that

$$
\mathscr{A}_{ \pm}=g \partial_{ \pm} g^{-1} \text {. }
$$

These two equations are compatible only if $\mathscr{A}$ satisfies the integrability condition

$$
\left[\partial_{+}+\mathscr{A}_{+}, \partial_{-}+\mathscr{A}_{-}\right]=0 \text {. }
$$

Working this out with the aid of the commutation relations (5) we find that $\varrho$ and $\phi$ must satisfy

$$
\begin{aligned}
& \partial^{2} \varrho+4 \alpha^{2} \sin \varrho=0, \\
& \partial^{2} \phi+\frac{\alpha^{2}}{2} e^{-i \varrho}=0 .
\end{aligned}
$$

The first equation is the sine-Gordon equation we are interested in, whilst the second fixes the auxiliary field $\phi$ in terms of $\varrho$ up to the addition of a solution to the free wave equation.

Our method for solving the sine-Gordon equation derives from studying the action of $g$ on two particular vectors in the representation space of the Kac-Moody algebra. The first vector, $|\Lambda\rangle$, corresponds to a fundamental dominant weight, i.e. it satisfies

$$
H_{0}|\Lambda\rangle=|\Lambda\rangle, \quad \mathbb{1}|\Lambda\rangle=|\Lambda\rangle, \quad E_{0}^{+}|\Lambda\rangle=0, \quad L_{n}^{i}|\Lambda\rangle=0 \quad \text { for } \quad n>0,
$$

together with conjugate equations

$$
\langle\Lambda| H_{0}=\langle\Lambda|, \quad\langle\Lambda| \mathbb{1}=\langle\Lambda|, \quad\langle\Lambda| E_{0}^{-}=0, \quad\langle\Lambda| L_{n}^{i}=0 \quad \text { for } \quad n<0 .
$$

The vector $|\Lambda\rangle$ is a generalisation of the column vector $(1,0)^{T}$ on which the Lie algebra acts. The second vector we need is a generalisation of the trivial representation of the Lie algebra and satisfies

$$
\begin{aligned}
& L_{n}^{i}|0\rangle=0, \quad n \geqq 0, \\
& \mathbb{1}|0\rangle=|0\rangle, \\
& \langle 0| L_{n}^{i}=0, \quad n \leqq 0, \\
& \langle 0| \mathbb{1}=\langle 0| .
\end{aligned}
$$

For the moment we will assume the existence of vectors with these properties, but in the next section we will construct them. The solution of the sine-Gordon equation follows from observing that

$$
\partial_{+}\left\{g^{-1} e^{-(i \varrho / 4+\phi)}\right\}|\Lambda\rangle=g^{-1} e^{-(i \varrho / 4+\phi)}\left\{\mathscr{A}_{+}-\frac{1}{4} \partial_{+} i \varrho-\partial_{+} \phi\right\}|\Lambda\rangle=0,
$$

and

$$
\partial_{-}\left\{\langle\Lambda| e^{-(i \varrho / 4+\phi)} g\right\}=\langle\Lambda|\left\{-\mathscr{A}_{-}-\frac{1}{4} \partial_{-} i \varrho-\partial_{-} \phi\right\} e^{-(i \varrho / 4+\phi)} g=0,
$$

using the properties of $|\Lambda\rangle$. Similarly,

Thus the quantity

$$
\partial_{+}\left\{g^{-1} e^{-\phi}\right\}|0\rangle=0, \quad \partial_{-}\left\{\langle 0| e^{-\phi} g\right\}=0 .
$$

$$
\exp -\left(\frac{1}{4} i[\varrho(A)+\varrho(B)]+\phi(A)+\phi(B)\right)\left\langle\Lambda\left|g(A) g^{-1}(B)\right| \Lambda\right\rangle
$$


is independent of both the $Z^{-}$coordinate of the point $A$ and the $Z^{+}$coordinate of the point $B$. This enables us to evaluate it firstly by choosing these coordinates so that $A$ and $B$ coincide, at the point $P$ say, where it is simply

$$
\exp -\left(\frac{1}{2} i \varrho(P)+2 \phi(P)\right)
$$

and secondly by choosing them so that $A$ and $B$ lie on $t=0$, in which case we construct $g(A) g^{-1}(B)$ from a knowledge of the values of the fields $\varrho$ and $\phi$ on $t=0$. Although the values of $\varrho$ on $t=0$ constitute our initial data we do not know $\phi$, but we will be able to eliminate it from the problem later on. If $P$ has the coordinates $(x, t)$, then $A$ is the point $(x+t, 0)$ and $B$ is $(x-t, 0)$. From (6) and (8) we have

$$
\begin{aligned}
g \frac{\partial}{\partial x} g^{-1} & =\frac{1}{2}\left(\mathscr{A}_{+}-\mathscr{A}_{-}\right) \\
& =\frac{1}{4} i \dot{\varrho} H_{0}+\frac{\alpha}{2} e^{i \varrho / 2}\left(E_{0}^{+}-E_{0}^{-}\right)+\frac{\alpha}{2} e^{-i \varrho / 2}\left(E_{1}^{-}-E_{-1}^{+}\right)+\phi \mathbb{1} \\
& \equiv \mathscr{H}+\phi \dot{\mathbb{1}}
\end{aligned}
$$

(the dots denote differentiation with respect to time). So that $g(A) g^{-1}(B)$ may be calculated as a path ordered exponential integral from $B$ to $A$ along $t=0$,

$$
\begin{aligned}
g(A) g^{-1}(B) & =P \exp -\int_{B}^{A} d x(\mathscr{H}+\phi \mathbb{1}) \\
& =\left(\exp -\int_{B}^{A} d x \phi\right) P \exp -\int_{B}^{A} d x \mathscr{H} .
\end{aligned}
$$

Putting all this together gives

$$
\begin{aligned}
\exp & -\left(\frac{1}{2} i \varrho(P)+2 \phi(P)\right) \\
= & \exp -\left(\frac{1}{4} i[\varrho(A)+\varrho(B)]+\phi(A)+\phi(B)+\int_{B}^{A} d x \phi\right) \\
\cdot & \left\langle\Lambda\left|P \exp -\int_{B}^{A} d x \mathscr{H}\right| \Lambda\right\rangle .
\end{aligned}
$$

By a similar argument based on Eq. (17) we find that

$$
\begin{aligned}
\exp -2 \phi(P)= & \exp -\left(\phi(A)+\phi(B)+\int_{B}^{A} d x \phi\right) \\
& \cdot\left\langle 0\left|P \exp -\int_{B}^{A} d x \mathscr{H}\right| 0\right\rangle .
\end{aligned}
$$

Dividing (22) by (23) eliminates the field $\phi$ from our expressions and yields the solution to the initial value problem for the sine-Gordon equation in the form

$$
\exp -\frac{1}{2} i \varrho(P)=\exp \left(-\frac{1}{4} i[\varrho(A)+\varrho(B)]\right) \frac{\left\langle\Lambda\left|P \exp -\int_{B}^{A} d x \mathscr{H}\right| \Lambda\right\rangle}{\left\langle 0\left|P \exp -\int_{B}^{A} d x \mathscr{H}\right| 0\right\rangle} .
$$


In principle this solves the sine-Gordon equation because it expresses the field at the point $P$ in terms of its values at time $t=0$. All that is needed to evaluate the matrix elements appearing in this expression is a knowledge of the commutation relations for the algebra (5) together with the defining properties of $|\Lambda\rangle$ and $|0\rangle$ (12)-(14). However, this is a very cumbersome way to proceed and offers little insight into the structure of the solution. A better approach is suggested by the obvious resemblance of $(24)$ to a quantum mechanical Green function for a system evolving in the "time" variable $x$ with Hamiltonian $-i \mathscr{H}$. In the next section we describe a fermionic representation of the Kac-Moody algebra which will make this precise. We will then be able to evaluate the right-hand side of (24) using Wick's theorem.

\section{Fermionic Representation of Kac-Moody Algebras}

It has been known for some time that currents constructed from fermion fields can provide explicit realisations of the type of infinite dimensional algebras we have been using [10]. Here we outline one way of doing this. It is based on an $n$-component complex Fermi field $\psi_{r}(\sigma)$ defined on the space-like unit interval $0 \leqq \sigma \leqq 1$ with periodic boundary conditions and canonical anticommutation relations,

$$
\left\{\psi_{r}^{+}(\sigma), \psi_{s}\left(\sigma^{\prime}\right)\right\}=\delta_{r s} \delta\left(\sigma-\sigma^{\prime}\right), \quad\left\{\psi_{r}(\sigma), \psi_{s}\left(\sigma^{\prime}\right)\right\}=0 .
$$

The coefficients of the Fourier decomposition of $\psi$ have the usual creation and annihilation operator algebra that follows from (25),

$$
\psi_{r}(\sigma)=\sum_{n=-\infty}^{\infty} A_{n}^{r} e^{2 \pi i n \sigma}, \quad\left\{A_{n}^{r}, A_{m}^{s}\right\}=0, \quad\left\{A_{n}^{r+}, A_{m}^{s}\right\}=\delta^{r s} \delta_{n m} .
$$

Which of these operators we take to be annihilation operators and which creation operators appears at this stage to be rather arbitrary, but with some prejudice we will define our Fock space vacuum so that

$$
\begin{aligned}
& A_{n}^{r}|0\rangle=0, \quad n \geqq 0 \quad \text { all } r, \\
& A_{n}^{r+}|0\rangle=0, \quad n \leqq-1 \quad \text { all } r \text {. }
\end{aligned}
$$

The Fock space states are generated in the usual way, by applying the remaining operators to the vacuum. Conjugate to (27) we have

$$
\begin{array}{rll}
\langle 0| A_{n}^{r+}=0, & n \geqq 0 & \text { all } r, \\
\langle 0| A_{n}^{r}=0, & n \leqq-1 & \text { all } r .
\end{array}
$$

Matrix elements of products of the Fermi field will not be finite in general. However, normal ordered products will have finite matrix elements. In particular, the Fermion bilinears

$$
J^{i}(\sigma)=: \bar{\psi}(\sigma) L^{i} \psi(\sigma):
$$

will have a finite action on the Fock space. Here we have assembled the $\psi_{r}$ into a column vector $\psi$ and the $\psi_{r}^{+}$into a row vector $\bar{\psi}$. The $L^{i}$ are the $n \times n$ matrices introduced in Sect. 2 which form a representation of the Lie algebra. The normal 
ordering, which is denoted by dots, stands the annihilation operators to the right of the creation operators at the cost of a minus sign for every interchange of operators that takes (29) back to the ordinary product.

In the Appendix we show that the Fourier components of $J$ satisfy the KacMoody algebra. Thus if

$$
L_{n}^{i}=\int_{0}^{1} d \sigma e^{-2 \pi i n \sigma} J^{i}(\sigma),
$$

then

$$
\left[L_{n}^{i}, L_{m}^{j}\right]=f^{i j k} L_{m+n}^{k}+n \delta_{n,-m} \eta^{i j} \mathbb{1},
$$

where $\mathbb{1}$ is just the $c$-number one. Writing out $L_{n}^{i}$ in terms of the $A$ and $A^{+}$,

$$
L_{n}^{i}=\sum_{q}: A_{q-n}^{r+} L_{r s}^{i} A_{q}^{s}:
$$

we see that for $n \geqq 0$ each term in the sum contains at least one annihilation operator which stands to the right because of the normal ordering; thus

$$
L_{n}^{i}|0\rangle=0, \quad n \geqq 0 .
$$

Similarly,

$$
\langle 0| L_{n}^{i}=0, \quad n \leqq 0,
$$

so that the vacuum has all the properties we required of the vector $|0\rangle$ in Sect. 2 . We will identify them. We also need $|\Lambda\rangle$. If the action of the $n \times n$ matrix $L^{i}$ on the column vector $\phi$ is to give the column vector $\chi$,

$$
L^{i} \phi=\chi,
$$

then the action of the Kac-Moody generator $L_{0}^{i}$ on the state

$$
|\phi\rangle \equiv \sum_{r} \phi^{r} A_{0}^{r+}|0\rangle
$$

is to give the state $|\chi\rangle$, since if we use (26) and (32) it is easy to see that

$$
\left[L_{n}^{i}, A_{0}^{s+}\right]=\sum_{r} L_{r s}^{i} A_{-n}^{r+} .
$$

So that

$$
L_{0}^{i}|\phi\rangle=\sum_{s}\left[L_{0}^{i}, A_{0}^{s+}\right]|0\rangle \phi^{s}=\sum_{r s} L_{r s}^{i} A_{0}^{r+}|0\rangle \phi^{s}=|\chi\rangle .
$$

Also $L_{n}^{i}$ annihilates $|\phi\rangle$ if $n \geqq 1$, because

$$
L_{n}^{i}|\phi\rangle=\sum_{r} \chi^{r} A_{-n}^{r+}|0\rangle=0, \quad n \geqq 1 .
$$

The vector $|\Lambda\rangle$ introduced in Sect. 2 was a generalisation of $(1,0)^{T}$ in the same way that $|\phi\rangle$ generalises $\phi$. Thus we can take

$$
|\Lambda\rangle=A_{0}^{1+}|0\rangle=\int_{0}^{1} d \sigma \psi_{1}^{+}(\sigma)|0\rangle
$$

as this satisfies all the conditions (12). 


\section{The Solution in Terms of a Fermionic Propagator}

We can now translate our solution of the sine-Gordon equation into the language of Fermi fields of the previous section. The result will be to reduce our formula (24) to a Fermionic propagator which may be evaluated in the usual way as a sum of Feynman diagrams. The expression is particularly simple because the Hamiltonian is only bilinear in the Fermi fields. It may be written

$$
\begin{aligned}
\mathscr{H}(x)= & i \varrho(x, 0) H_{0}+\frac{\alpha}{2} e^{i \varrho(x, 0) / 2}\left(E_{0}^{+}-E_{0}^{-}\right) \\
& +\frac{\alpha}{2} e^{-\varrho(x, 0) / 2}\left(E_{1}^{-}-E_{-1}^{+}\right) \\
= & i \int d \sigma: \bar{\psi}(\sigma) M(\sigma, x) \psi(\sigma):,
\end{aligned}
$$

where $M$ is the $2 \times 2$ matrix

$M(\sigma, x)=\left[\begin{array}{cc}\frac{1}{4} \dot{\varrho}(x, 0) & \frac{i \alpha}{2}\left(-e^{i \varrho(x, 0) / 2}+e^{-i \varrho(x, 0) / 2} e^{2 \pi i \sigma}\right) \\ \frac{i \alpha}{2}\left(e^{i \varrho(x, 0) / 2}-e^{-i \varrho(x, 0) / 2} e^{-2 \pi i \sigma}\right) & -\frac{1}{4} \dot{\varrho}(x, 0)\end{array}\right]$.

In terms of the Fermi fields the ratio of matrix elements appearing in our solution becomes

$$
\begin{gathered}
\left\langle\Lambda\left|P \exp -\int_{B}^{A} d x \mathscr{H}\right| \Lambda\right\rangle /\left\langle 0\left|P \exp -\int_{B}^{A} d x \mathscr{H}\right| 0\right\rangle \\
=\int_{0}^{1} d \sigma_{A} d \sigma_{B} \widetilde{G}_{11}\left(\mathbf{x}_{A}, \mathbf{x}_{B}\right), \\
\widetilde{G}_{r s}\left(\mathbf{x}_{A}, \mathbf{x}_{B}\right)=\frac{\left\langle 0\left|\psi_{r}\left(\sigma_{A}\right) P \exp \left(-i \int d^{2} \mathbf{x}: \bar{\psi} M \psi:\right) \psi_{s}^{+}\left(\sigma_{B}\right)\right| 0\right\rangle}{\left\langle 0\left|P \exp \left(-i \int d^{2} \mathbf{x}: \bar{\psi} M \psi:\right)\right| 0\right\rangle} .
\end{gathered}
$$

Here the vector $\mathbf{x}$ is the pair $(x, \sigma)$. It is convenient to consider a slightly more general object than $\tilde{G}$. Define

so that

$$
G_{r s}\left(\mathbf{x}, \mathbf{x}^{\prime} ; \mathbf{x}_{A}, \mathbf{x}_{B}\right)=\frac{\left\langle 0\left|P \psi_{r}(\mathbf{x}) \exp \left(-i \int_{x_{B}}^{x_{A}} d \tilde{x} \int_{0}^{1} d \tilde{\sigma}: \bar{\psi} M \psi:\right) \psi_{s}^{+}(\mathbf{x})\right| 0\right\rangle}{\left\langle 0\left|P \exp \left(-i \int_{x_{B}}^{x_{A}} d \tilde{x} \int_{0}^{1} d \tilde{\sigma}: \bar{\psi} M \psi:\right)\right| 0\right\rangle}
$$

$$
. \widetilde{G}_{r s}\left(\mathbf{x}_{A}, \mathbf{x}_{B}\right)=G_{r s}\left(\mathbf{x}_{A}, \mathbf{x}_{B} ; \mathbf{x}_{A}, \mathbf{x}_{B}\right) .
$$

$\psi$ depends on $x$ only as on a label which determines its position under path ordering, otherwise it just depends on $\sigma . G$ is the two point function for a fermionic theory with Hamiltonian density : $\bar{\psi} M \psi:$, this is the result anticipated at the end of Sect. 2. Furthermore, because : $\bar{\psi} M \psi$ : contains no derivatives of the Fermi fields, the differential equation for which $G$ is a Green function will only involve 
derivatives with respect to the "time" variable $x$. Using Wick's theorem we obtain $G$ as the usual diagrammatic expansion of the complete propagator.

$$
\begin{aligned}
& G_{r s}\left(\mathbf{x}, \mathbf{y} ; \mathbf{x}_{A}, \mathbf{x}_{B}\right) \equiv \frac{r}{x} \longrightarrow \frac{s}{y}= \\
& \frac{r}{x-y}+\frac{r}{x \longrightarrow y}+\frac{s}{x \longrightarrow \cdot}+\frac{s}{y}+
\end{aligned}
$$

Here the bare propagator is

$$
\begin{aligned}
\frac{r \quad s}{x \quad y} & =\left\langle 0\left|P \psi_{r}\left(\mathbf{x}_{1}\right) \psi_{s}^{+}\left(\mathbf{x}_{2}\right)\right| 0\right\rangle \\
& =\delta_{r s}\left\{\theta\left(x_{1}-x_{2}\right) \kappa_{>}\left(\sigma_{1}-\sigma_{2}\right)-\theta\left(x_{2}-x_{1}\right) \kappa_{<}\left(\sigma_{1}-\sigma_{2}\right)\right\} \\
& \equiv \delta_{r s} \Delta\left(\mathbf{x}_{1}-\mathbf{x}_{2}\right) .
\end{aligned}
$$

$\kappa_{>}$and $\kappa_{<}$are projection operators onto the positive and nonpositive frequency components of functions of $\sigma$. Thus

$$
\kappa_{\gtrless} \circ e^{2 \pi i n \sigma} \equiv \int d \sigma_{1} \kappa_{\gtrless}\left(\sigma-\sigma_{1}\right) e^{2 \pi i n \sigma_{1}}= \begin{cases}e^{2 \pi i n \sigma} & n \geqq 0, \\ 0 & n<0, \\ 0 & \text { otherwise } .\end{cases}
$$

The vertex-— is just the matrix $-i M(\sigma, x)$. The implied integrations in (44) are over the ranges $0 \leqq \sigma \leqq 1, x_{B} \leqq x \leqq x_{A}$. Explicitly the expansion of the matrix $\left(G_{r s}\right)$ is

$$
\begin{aligned}
G\left(\mathbf{x}, \mathbf{y} ; \mathbf{x}_{A}, \mathbf{x}_{B}\right)= & \mathbb{1} \Delta(\mathbf{x}-\mathbf{y})-i \int d^{2} \mathbf{x}_{1} \Delta\left(\mathbf{x}-\mathbf{x}_{1}\right) M\left(\mathbf{x}_{1}\right) \Delta\left(\mathbf{x}_{1}-\mathbf{y}\right) \\
& +\int d^{2} \mathbf{x}_{1} d^{2} \mathbf{x}_{2} \Delta\left(\mathbf{x}-\mathbf{x}_{1}\right) M\left(\mathbf{x}_{1}\right) \Delta\left(\mathbf{x}_{1}-\mathbf{x}_{2}\right) M\left(\mathbf{x}_{2}\right) \Delta\left(\mathbf{x}_{2}-\mathbf{y}\right)+\ldots
\end{aligned}
$$

Rearranging (44) gives

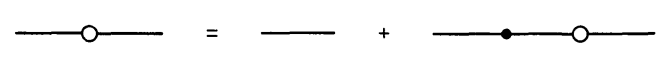

which together with

$$
\frac{\partial}{\partial x_{1}} \Delta\left(\mathbf{x}_{1}-\mathbf{x}_{2}\right)=\delta\left(x_{1}-x_{2}\right) \delta\left(\sigma_{1}-\sigma_{2}\right)
$$

shows that $G$ is a Green function

$$
\left(\frac{\partial}{\partial x_{1}}+i M\left(\mathbf{x}_{1}\right)\right) G\left(\mathbf{x}_{1}, \mathbf{x}_{2} ; \mathbf{x}_{A}, \mathbf{x}_{B}\right)=\delta\left(x_{1}-x_{2}\right) \delta\left(\sigma_{1}-\sigma_{2}\right) .
$$

This is the Green function appropriate to "mixed" or Feynman type boundary conditions that are usual in relativistic quantum field theory. By this we mean that

$$
\phi(\mathbf{x})=\int d \sigma_{B} G\left(\mathbf{x}, \mathbf{x}_{B} ; \mathbf{x}_{A}, \mathbf{x}_{B}\right) \tilde{\phi}_{>}\left(\sigma_{B}\right)-\int d \sigma_{A} G\left(\mathbf{x}, \mathbf{x}_{A} ; \mathbf{x}_{A}, \mathbf{x}_{B}\right) \tilde{\phi}_{<}\left(\sigma_{A}\right)
$$

satisfies

$$
\left(\frac{\partial}{\partial x}+i M(\mathbf{x})\right) \phi(\mathbf{x})=0,
$$

subject to

$$
\kappa_{>} \circ \phi\left(x_{B}, \sigma_{B}\right)=\tilde{\phi}_{>}\left(\sigma_{B}\right), \quad \kappa_{<} \circ \phi\left(x_{A}, \sigma_{A}\right)=\tilde{\phi}_{<}\left(\sigma_{A}\right) .
$$


This may be seen by solving (52) and (53) iteratively in powers of $M$. We can now interpret the right-hand side of (42) in terms of such a $\phi$,

where

$$
\int_{0}^{1} d \sigma_{A} d \sigma_{B} \widetilde{G}_{11}\left(\mathbf{x}_{A}, \mathbf{x}_{B}\right)=\int d \sigma_{B}(1,0) \phi\left(x_{B}\right)
$$

$$
\begin{aligned}
\left(\frac{\partial}{\partial x}+i M(\mathbf{x})\right) \phi(\mathbf{x}) & =0, \\
\kappa_{>} \circ \phi\left(\mathbf{x}_{B}\right) & =\left(\begin{array}{l}
1 \\
0
\end{array}\right), \\
\kappa_{<} \circ \phi\left(\mathbf{x}_{A}\right) & =0 .
\end{aligned}
$$

Now we can give the final form of our solution of the initial value problem of the sine-Gordon equation.

$$
\exp -\frac{1}{2} i \varrho(x, t)=\exp \left(-\frac{1}{4} i[\varrho(x+t, 0)+\varrho(x-t, 0)]\right) \int d \sigma_{A}(1,0) \phi\left(\mathbf{x}_{A}\right),
$$

where $\phi$ satisfies (54) and the $2 \times 2$ matrix $M$ contains the initial data. The solution of (54) is given by (51) in conjunction with (47).

\section{Relationship to the Inverse Scattering Method}

In this section we discuss the relationship of our work to the Riemann transform method of Shabat et al. [7]. This is a version of the inverse scattering transform in which solutions of the sine-Gordon equation are constructed by solving a Riemann problem. The problem is to construct a $2 \times 2$ matrix function $\psi$ of the complex variable $\lambda$ which is analytic everywhere except on some contour $\Gamma$ where it suffers a discontinuity. The limiting values of $\psi$ on either side of the contour are determined by the conjugation condition

$$
\psi_{>}(\lambda)=G(\lambda) \psi_{<}(\lambda), \quad \lambda \in \Gamma,
$$

together with the value of $\psi$ at some point

$$
\left.\psi(\lambda)\right|_{\lambda=\infty}=\mathbb{1} .
$$

Both $\psi$ and the matrix $G$ are functions of the spacetime variables $x$ and $t$, and $G$ satisfies

$$
\left[\partial_{ \pm}+\tilde{\mathscr{A}}_{ \pm}, G\right]=0 .
$$

$\left\{\tilde{\mathscr{A}}_{ \pm}\right\}$is a Lax pair depending on the spectral parameter $\lambda$, whose zero curvature condition

$$
\left[\partial_{+}+\tilde{\mathscr{A}}_{+}, \partial_{-}+\tilde{\mathscr{A}}_{-}\right]=0
$$

is equivalent to the sine-Gordon equation in the same way that (9) leads to (10). The Riemann problem is solved by using Cauchy's integral formula to write down an integral equation for $\psi_{>}$in terms of $G$, which may then be solved by iteration, see [7] for details.

We now want to connect this with our work. We do so by showing that the defining equations for $\phi(54)$ may be cast as a similar Riemann problem, which in our case is solved by (51) together with the Wick expansion (47). The $\phi$ of (54) is one 
column of a $2 \times 2$ matrix $\Phi$ satisfying

$$
\begin{gathered}
\left(\frac{\partial}{\partial x}+i M(x, \zeta)\right) \Phi=0, \quad \zeta=e^{2 \pi i \sigma}, \\
\kappa_{>} \circ \Phi\left(\mathbf{x}_{B}\right)=\mathbb{1}, \quad \kappa_{<} \circ \Phi\left(\mathbf{x}_{A}\right)=0 .
\end{gathered}
$$

We can readily write down a formal solution to the differential equation,

$$
\Phi\left(\mathbf{x}_{A}\right)=G\left(x_{A}, x_{B}, \zeta\right) \Phi\left(\mathbf{x}_{B}\right), \quad G=P \exp -i \int_{x_{B}}^{x_{A}} d x M(x, \zeta)
$$

The problem is to satisfy the boundary conditions. These force $\Phi\left(\mathbf{x}_{A}\right)$ to be a function not just of $x_{A}$ but of $x_{B}$ also, and similarly for $\Phi\left(\mathbf{x}_{B}\right)$, so define

$$
\Phi\left(\mathbf{x}_{A}\right)=\phi_{>}\left(x_{A}, x_{B}, \zeta\right), \quad \Phi\left(\mathbf{x}_{B}\right)=\phi_{<}\left(x_{A}, x_{B}, \zeta\right) .
$$

$\phi_{>}$and $\phi_{<}$are both periodic functions of $\sigma$, and so have power series expansions in $\zeta$. The boundary condition

$$
\kappa_{>} \circ \phi_{<}=\mathbb{1}
$$

implies that there are no positive powers of $\zeta$ in this expansion, so we may continue this expansion to define $\phi_{<}$as an analytic function of $\zeta$ for $|\zeta|>1$ such that

$$
\underset{\zeta \rightarrow \infty}{\operatorname{Lt}} \phi_{<}(\zeta)=\mathbb{1}
$$

Similarly, the boundary condition

$$
\kappa_{<} \circ \phi_{>}=0
$$

implies that the power series expansion for $\phi_{>}$may be analytically continued to define an analytic function for $|\zeta|<1$. The two analytic functions are, of course, related by (61) on the unit circle

$$
\phi_{>}(\zeta)=G(\zeta) \phi_{<}(\zeta), \quad|\zeta|=1
$$

The dependence of $G$ on the space-time variable $x$ is through $x_{A}$ and $x_{B}$

$$
x_{A}=x+t, \quad x_{B}=x-t,
$$

so that

$$
\frac{\partial}{\partial x} G=-i M\left(x_{A}\right) G+G i M\left(x_{B}\right)
$$

$i M$ is the spatial component of a Lax pair,

$$
\tilde{\mathscr{A}}_{ \pm}= \pm \frac{i}{4} \partial_{ \pm} \varrho H+\alpha e^{i \varrho / 2} E^{ \pm}+\alpha \zeta^{\mp 1} e^{-i \varrho / 2} E^{\mp}
$$

whose zero curvature condition yields the sine-Gordon equation. The Eqs. (68) and (58) differ in the arguments of $M$; this is because our $G$ contains only the initial values of the sine-Gordon field as we have expressed the time development through our solution (55). 


\section{Conclusion}

We have solved the initial value problem for the classical sine-Gordon equation by a Lax pair technique based on a Kac-Moody algebra. Realising the algebra in terms of Fermionic currents reduced the problem to that of evaluating a propagator for the Fermi fields for which there exists the standard expansion as a sum of Feynman diagrams. The advantage of the method over the inverse scattering transform is that it can be performed for arbitrary initial data, although in general the Feynman diagrams could probably only be summed numerically rather than in closed form.

That the classical model reduces to a Fermionic theory is intriguing because of the well-known equivalence between the quantum version and the massive Thirring model. Attempting to apply our method to the quantum sine-Gordon equation along the lines of [11] would presumably reproduce this equivalence.

Finally, we should point out that, independently of the present work, Olive and Turok [12] have considered the construction of solutions to the sine-Gordon equation and its generalisations within the context of a Lax pair belonging to a Kac-Moody algebra. They are interested in the hierarchy of equations based on the two dimensional exactly integrable systems obtained by replacing the algebra $\operatorname{sl}(2, \mathbb{C})$ used here by an arbitrary Lie algebra.

\section{Appendix}

In this appendix we show that the $L_{n}^{i}$ defined in Eq. (30) satisfy the Kac-Moody algebra. We begin by using the $\left\{A_{n}^{r}\right\}$ defined in (26) to construct "analytic continuations" of the Fermi fields for complex $Z$

$$
\phi_{r}(Z)=\sum_{n=-\infty}^{\infty} A_{n}^{r} Z^{n}, \quad \bar{\phi}_{r}(Z)=\sum_{n=-\infty}^{\infty} A_{n}^{r+} Z^{-n} .
$$

Splitting each of these into creation and annihilation parts

$$
\begin{array}{cc}
\phi_{r}(Z)=C_{r}(Z)+A_{r}(Z), & \bar{\phi}_{r}(Z)=\bar{A}_{r}(Z)+\bar{C}_{r}(Z), \\
C_{r}(Z)=\sum_{n \leqq-1} A_{n}^{r} Z^{n}, & \bar{C}_{r}(Z)=\sum_{n \leqq-1} A_{n}^{r+} Z^{-n}, \\
A_{r}(Z)=\sum_{n \geqq 0} A_{n}^{r} Z^{n}, & \bar{A}_{r}(Z)=\sum_{n \geqq 0} A_{n}^{r+} Z^{-n},
\end{array}
$$

enables us to write out the normal ordered product

$$
: \bar{\phi}_{r}(Z) \phi_{s}(Z):=-C_{s} \bar{C}_{r}+\bar{C}_{r} A_{s}+\bar{A}_{r} C_{s}+\bar{A}_{r} A_{s} .
$$

The anticommutators of the annihilation and creation parts of $\phi\left(Z_{1}\right)$ and $\bar{\phi}\left(Z_{2}\right)$ are well defined functions of $Z_{1}$ and $Z_{2}$ only for certain values of $\left|Z_{1} / Z_{2}\right|$ :

$$
\begin{aligned}
\left\{C_{r}\left(Z_{1}\right), \bar{C}_{s}\left(Z_{2}\right)\right\} & =\delta_{r s} \sum_{n \geqq 1}\left(Z_{2} / Z_{1}\right)^{n} \\
& =\delta_{r s} Z_{2} /\left(Z_{1}-Z_{2}\right) \text { for }\left|Z_{2}\right|<\left|Z_{1}\right|, \\
\left\{A_{r}\left(Z_{1}\right), \bar{A}_{s}\left(Z_{2}\right)\right\} & =\delta_{r s} \sum_{n \geqq 0}\left(Z_{1} / Z_{2}\right)^{n} \\
& =\delta_{r s} Z_{2} /\left(Z_{2}-Z_{1}\right) \text { for }\left|Z_{1}\right|<\left|Z_{2}\right| .
\end{aligned}
$$


Consider the following product,

$$
: \bar{\phi}_{r}\left(Z_{1}\right) \phi_{s}\left(Z_{1}\right):: \bar{\phi}_{t}\left(Z_{2}\right) \phi_{u}\left(Z_{2}\right): \text {. }
$$

For $\left|Z_{1}\right|<\left|Z_{2}\right|$ a little algebra allows this to be rewritten as

$$
\begin{aligned}
& : \bar{\phi}_{r}\left(Z_{1}\right) \phi_{s}\left(Z_{1}\right) \bar{\phi}_{t}\left(Z_{2}\right) \phi_{u}\left(Z_{2}\right):+\delta_{s t} \frac{Z_{2}}{Z_{2}-Z_{1}}: \bar{\phi}_{r}\left(Z_{1}\right) \phi_{u}\left(Z_{2}\right): \\
& \quad-\delta_{r u} \frac{Z_{1}}{Z_{2}-Z_{1}}: \bar{\phi}_{t}\left(Z_{2}\right) \phi_{s}\left(Z_{1}\right):+\delta_{s t} \delta_{r u} Z_{1} Z_{2} /\left(Z_{2}-Z_{1}\right)^{2} \\
& =\Gamma_{r s t u}\left(Z_{1}, Z_{2}\right) .
\end{aligned}
$$

The value of this expression is that all the operator products are normal ordered and so have a finite action on the Fock space, even as $Z_{1}$ approaches $Z_{2}$. Now define

$$
E_{r s}^{n}=\int_{0}^{1} d \sigma e^{-2 \pi i n \sigma}: \psi_{r}^{+}(\sigma) \psi_{s}(\sigma)
$$

In terms of $\phi$ and $\bar{\phi}$ this is

$$
E_{r s}^{n}=\frac{1}{2 \pi i} \oint_{c} \frac{d Z}{Z^{n+1}}: \bar{\phi}_{r}(Z) \phi_{s}(Z):
$$

$c$ is the unit circle. Using (A.6) the product of two E's is

$$
E_{r s}^{n} E_{t u}^{m}=\frac{1}{(2 \pi i)^{2}} \oint_{c_{1}} \frac{d Z_{1}}{Z_{1}^{n+1}} \oint_{c_{2}} \frac{d Z_{2}}{Z_{2}^{m+1}} \Gamma_{r s t u}\left(Z_{1}, Z_{2}\right) .
$$

$c_{1}$ is a closed contour lying just within the unit circle whilst $c_{2}$ just encircles it. This ensures that $\left|Z_{1}\right|<\left|Z_{2}\right|$. Similarly,

$$
E_{t u}^{m} E_{r s}^{n}=\frac{1}{(2 \pi i)^{2}} \oint_{c_{1}^{\prime}} \frac{d Z_{1}}{Z_{1}^{n+1}} \oint_{c_{2}} \frac{d Z_{2}}{Z_{2}^{m+1}} \Gamma_{r s t u}\left(Z_{1}, Z_{2}\right) .
$$

The integrands are the same because of the symmetry of $\Gamma$ under the interchange of $\left(r, s, Z_{1}\right)$ and $\left(t, u, Z_{2}\right)$, but now $c_{1}^{\prime}$ must encircle $c_{2}$ so that $\left|Z_{1}\right|>\left|Z_{2}\right|$, the order of the $E$ 's having been reversed. Subtracting (A.10) from (A.9),

$$
\left[E_{r s}^{n}, E_{t u}^{m}\right]=\frac{1}{(2 \pi i)^{2}} \oint_{c_{2} c_{1}-c_{1}^{\prime}} \frac{d Z_{1}}{Z_{1}^{n+1}} \frac{d Z_{2}}{Z_{2}^{m+1}} \Gamma_{r s t u}\left(Z_{1}, Z_{2}\right) .
$$

Do the $Z_{1}$ integration first, holding $Z_{2}$ fixed. The contour $c_{1}-c_{1}^{\prime}$ can be deformed to a small circle around the pole $Z_{1}=Z_{2}$ of $\Gamma$, traversed clockwise. Thus

$$
\begin{aligned}
{\left[E_{r s}^{n}, E_{t u}^{m}\right]=} & \frac{1}{2 \pi i} \oint_{c_{2}} \frac{d Z_{2}}{Z_{2}^{m+n+1}} \\
& \cdot\left\{\delta_{s t}: \bar{\phi}_{r}\left(Z_{2}\right) \phi_{u}\left(Z_{2}\right):-\delta_{r u}: \bar{\phi}_{t}\left(Z_{2}\right) \phi_{s}\left(Z_{2}\right):+n \delta_{r u} \delta_{s t}\right\} \\
= & E_{r u}^{n+m} \delta_{s t}-E_{t s}^{n+m} \delta_{r u}+n \delta_{r u} \delta_{s t} \delta_{n,-m} .
\end{aligned}
$$

The $n \times n$ matrices $\left\{L^{i} ; i=1 \ldots D\right\}$ satisfy the Lie algebra

$$
\left[L^{i}, L^{j}\right]=f^{i j k} L^{k}
$$


with Killing form

Define

$$
\eta^{i j}=\operatorname{Tr}\left(L^{i} L^{j}\right)
$$

$$
L_{n}^{i}=E_{r s}^{n} L_{r s}^{i}=\int_{0}^{1} d \sigma e^{-2 \pi i n \sigma}: \psi^{+}(\sigma) L^{i} \psi(\sigma):
$$

By contracting (A.12) with $L_{r s}^{i} L_{t u}^{j}$ we see that the $L_{n}^{i}$ satisfy the Kac-Moody algebra

$$
\left[L_{n}^{i}, L_{m}^{j}\right]=f^{i j k} L_{m+n}^{k}+n \delta_{n,-m} \eta^{i j} .
$$

\section{References}

1. Scott, A.C.: Propagation of magnetic flux on a long Josephson tunnel junction. Nuovo Cimento 69 B, 241 (1970);

Rubinstein, J.: Sine-Gordon equation. J. Math. Phys. (N.Y.) 11, 258 (1970)

Barone, A., et al.: Theory and applications of the sine-Gordon equation. Riv Nuovo Cimento 1, 227 (1971)

2. Ablowitz, M.J., et al.: Method for solving the sine-Gordon equation. Phys. Rev. Lett. 30, 1262 (1973)

3. Coleman, S.: Quantum sine-Gordon equation as a massive Thirring model. Phys. Rev. D 11, 2088 (1975)

Mandelstam, S.: Soliton operators for the quantized sine-Gordon equation. Phys. Rev. D 11, 3026 (1975)

4. Leznov, A.N., Smirnov, V.G.: Graded algebras of the second rank and integration of nonlinear equations $Y_{z \bar{z}}=\exp (2 Y)-\exp (-2 Y), Y_{z \bar{z}}=2 \exp (Y)-\exp (-2 Y)$. Lett. Math. Phys. 5, 31 (1981)

5. Leznov, A.N., Saveliev, M.V.: Representation of zero curvature for the system of nonlinear partial differential equations $X_{\alpha, z \bar{z}}=\exp (K X)_{\alpha}$ and its integrability. Lett. Math. Phys. 3, 489 (1979); Representation theory and integration of nonlinear spherically symmetric equations to gauge theory. Commun Math. Phys. 74, 111 (1980)

6. Onofri, E.: An identity for $T$-ordered exponentials with applications to quantum mechanics. Ann. Phys. 102, 371 (1976)

7. Mikhailov, A.V.: TH 3194 - CERN

Zakharov, V.E., Shabat, A.B.: Funct. Anal. Appl. 13, 13 (1979)

8. Jimbo, M., Miwa, T.: RIMS - 439

9 Kac, V.G.: Izv. Akad. Nauk. SSSR, Ser. Mat. 32, 1323 (1968); 34, 385 (1970)

Moody, R.V.: Bull. Am. Math. Soc. 73, 217 (1967)

10. Bardacki, K., Halpern, M.B.: New dual quark models. Phys. Rev. D 3, 2493 (1971)

Goddard, P., Olive, D.: Proceedings of the workshop on vertex operators in mathematics and physics. Berkeley, 1983

11. Mansfield, P.: Solution of Toda systems. Nucl. Phys. B 208, 277 (1982); Light-cone quantisation of the Liouville and Toda field theories. Nucl. Phys. B 222, 419 (1983)

12. Olive, D., Turok, N.: To appear

Communicated by A. Jaffe

Received July 30, 1984 
